\title{
A HIT-trapping strategy for rapid generation of reversible and conditional alleles using a universal donor
}

\author{
Hengxing Lu, ${ }^{1,4}$ Jun Liu, ${ }^{1,4}$ Tao Feng, ${ }^{2}$ Zihang Guo, ${ }^{1}$ Yunjun Yin, ${ }^{1}$ Fei Gao, ${ }^{1}$ \\ Gengsheng $\mathrm{Cao}^{3}{ }^{3}$ Xuguang $\mathrm{Du},{ }^{1}$ and Sen $\mathrm{Wu}^{1}$ \\ ${ }^{1}$ State Key Laboratory of Agrobiotechnology, College of Biological Sciences, China Agricultural University, Beijing 100193, China; \\ ${ }^{2}$ Institute of Urban Agriculture, Chinese Academy of Agricultural Sciences, Chengdu 610200, China; ${ }^{3}$ Henan Engineering Laboratory \\ for Mammary Bioreactor, School of Life Science, Henan University, Kaifeng 475004, China
}

\begin{abstract}
Targeted mutagenesis in model organisms is key for gene functional annotation and biomedical research. Despite technological advances in gene editing by the CRISPR-Cas9 systems, rapid and efficient introduction of site-directed mutations remains a challenge in large animal models. Here, we developed a robust and flexible insertional mutagenesis strategy, homology-independent targeted trapping (HIT-trapping), which is generic and can efficiently target-trap an endogenous gene of interest independent of homology arm and embryonic stem cells. Further optimization and equipping the HIT-trap donor with a site-specific DNA inversion mechanism enabled one-step generation of reversible and conditional alleles in a single experiment. As a proof of concept, we successfully created mutant alleles for 21 disease-related genes in primary porcine fibroblasts with an average knock-in frequency of $53.2 \%$, a great improvement over previous approaches. The versatile HIT-trapping strategy presented here is expected to simplify the targeted generation of mutant alleles and facilitate large-scale mutagenesis in large mammals such as pigs.
\end{abstract}

[Supplemental material is available for this article.]

Following the completion of animal genome sequencing projects, rapid and efficient mutagenesis strategies are needed for analyzing gene function and for creating human disease models. Gene trapping is a high-throughput mutagenesis strategy whereby random vector insertion can be achieved across the mouse genome. A typical gene-trap vector contains a promoter-less reporter/selection gene flanked by an upstream splice acceptor (SA) and a downstream poly(A) signal. Upon insertion into an intron of a gene, the vector both inactivates the trapped gene and enables the gene-specific expression of a reporter gene (Gossler et al. 1989; Stanford et al. 2001). To date, gene-trapping approaches have been successfully applied toward large-scale mutagenesis in mouse embryonic stem cells (mESCs) and generation of gene knockout mice (Skarnes et al. 2004). The main drawback of random gene trapping is that gene-trap alleles are not specifically engineered to target genes of interest in advance. Therefore, methods to streamline the introduction of predesigned, site-specific modifications into the genome by homologous recombination would represent a significant technological advance. Previously, a hybrid approach combining gene targeting and gene trapping (targeted trapping) enabled mutation of expressed genes in mESCs with high efficiency, using a gene-trap construct flanked by homologous sequences of the target locus (Friedel et al. 2005). Also, homologous recombination is commonly used for creating conditional alleles, which is essential to avoid embryonic lethality and to study the stage- and tissue-specific functions of genes (Branda and Dymecki 2004). However, both standard gene trap-

\footnotetext{
${ }^{4}$ These authors contributed equally to this work. Corresponding author: swu@cau.edu.cn

Article published online before print. Article, supplemental material, and publication date are at https://www.genome.org/cgi/doi/10.1101/gr.271312.120.
}

ping and targeted trapping are only suitable for genes expressed in embryonic stem (ES) cells. Furthermore, construction of targeting donor vectors with homology arms is labor intensive and costly, and the low efficiency of homologous recombination is also a rate-limiting step for gene targeting in mammalian genomes.

Recently, by taking advantage of precise genomic doublestrand breaks (DSBs) created by the clustered regularly interspaced short palindromic repeats (CRISPR)-associated protein 9 (Cas9) system (Ran et al. 2013; Doudna and Charpentier 2014; Hsu et al. 2014), homology-directed repair (HDR) efficiency was substantially enhanced (Porteus and Carroll 2005), and even donors with short homology arms (Orlando et al. 2010) or single-stranded DNA oligonucleotides (Chen et al. 2011; Quadros et al. 2017) were found to be compatible with site-specific integration. However, each targeting donor for HDR still needs to be customized with gene-specific homology sequences. Because of the lack of ES cells for certain animals such as pigs, sheep, and cattle, the genome must be edited either in a zygote embryo or in a somatic cell for somatic cell nuclear transfer (SCNT) (Reddy et al. 2020). It is still not feasible to achieve large-scale insertional mutagenesis including conditional knockouts in these important species with random gene trapping or HDR-based methods. Also, the problem of genetic mosaicism in embryo editing remains unresolved (Mehravar et al. 2019), prompting a need for technological advances to accelerate genetic modification in somatic cells.

(C) $2021 \mathrm{Lu}$ et al. This article is distributed exclusively by Cold Spring Harbor Laboratory Press for the first six months after the full-issue publication date (see https://genome.cshlp.org/site/misc/terms.xhtml). After six months, it is available under a Creative Commons License (Attribution-NonCommercial 4.0 International), as described at http://creativecommons.org/licenses/ by-nc/4.0/. 
Alternatively, the generally more efficient nonhomologous end joining (NHEJ) pathway has been exploited for site-specific insertion of exogenous DNA by simultaneous cleavage of both donor plasmid and genome using programmable nucleases (Cristea et al. 2013; Maresca et al. 2013; Brown et al. 2016; Suzuki et al. 2016; Sawatsubashi et al. 2018). In contrast to HDR-based strategies, NHEJ-mediated insertions do not require gene-specific homology arms, enabling diverse sites to be targeted with a universal donor vector. Therefore, we speculated that a gene-trap cassette could be inserted into a specific locus easily through this mechanism in any cell type.

Here, by combining NHEJ-mediated knock-in and gene trapping, we developed a strategy for targeted mutagenesis, especially in somatic cells with low HDR activity, referred to as HIT-trapping. By using a universal donor, this strategy allows us to (1) create null alleles, (2) produce a fluorescent reporter signal that could potentially allow cells with null alleles to be identified very quickly, and (3) produce reversible and conditional alleles that would be very helpful to have in most animal models but are often cumbersome to create.

\section{Results}

\section{Establishment of a CRISPR HIT-trapping method}

We designed a modular HIT-trapping system that uses the NHEJ pathway for targeting and trapping endogenous genes. Briefly, this system consists of three components: Cas9 protein, sgRNA expressing plasmids, and a universal gene-trap donor (HIT-trap-1C). Within the HIT-trap donor, an SA sequence followed by a promoter-less IRES-GFP reporter and a poly(A) signal (SA-IRES-GFP-pA) serves as a gene-trap cassette; a selection cassette constitutively expressing the puromycin-resistance gene (SV40-Puro-pA) is used to enrich target-trapped cells; and a RNU6-1 promoter expressing a sgRNA (termed sgA; against tia1l in zebrafish) is used to target the donor itself (Lackner et al. 2015). We placed the sgA recognition site between the RNU6-1-sgA and the gene-trap cassette that allows Cas9/sgA ribonucleoproteins (RNPs) to cleave the donor DNA in vivo. With concurrent cleavage of the donor plasmid and the selected targeting site by Cas9 RNPs, the NHEJ repair mechanism is expected to result in HIT-trap donor integration at the genomic DSB site. The inserted gene-trap cassette then generates a loss-of-function mutation and reports the expression of the target gene (Fig. 1A).

The HIT-trapping method was first examined by targeting the X-linked Hprt gene in XY mESCs, because targeting events of Hprt can be rapidly and stringently tested through 6-thioguanine (6-TG) treatment in culture (Doetschman et al. 1987). We thus generated three sgRNAs (sg-mTh1-3) against the first intron of Hprt (Supplemental Fig. S1A). The donor (HIT-trap-1C) and Cas9-expressing plasmid (pM3-Cas9) were cotransfected with sg-mTh1, $\mathrm{mTh} 2$, or mTh3 into mESCs and followed by puromycin selection. GFP-positive clones (Fig. 1B) were selected and expanded for genotyping by genome-donor junction PCR and Sanger sequencing. The results showed that we were able to obtain multiple correct target-trapped clones at all of the selected positions (Fig. 1C), albeit indels were created around the junctions (Supplemental Fig. S1B). Also, we obtained target-trapped clones at the Sall4 and Tet1 loci by identifying GFP-positive clones using the HIT-trapping approach (Supplemental Fig. S2A,B). In summary, the average efficiency of target-insertion of the HIT-trap donor was $51.6 \%$ at these three loci in mESCs (Supplemental Fig. S2C). We were able to obtain target-trapping events from a small number of clones (no more than nine clones per target site), indicating that most of the GFP-positive clones were correct knock-ins of the HIT-trap donors.

To assess the mutagenicity of the HIT-trap insertions, HITtrap clones Th1-1, Th2-4, and Th3-5 were subjected to qPCR, western blot, and toxicity analyses. In parallel, we also generated conventional knockout clones bearing indels at exon 2 (indel clones hk2/8/12) (Supplemental Fig. S1B) as a control group. Results showed that both strategies could efficiently abolish Hprt protein expression (Fig. 1D) and confer 6-TG resistance to the cells (Supplemental Fig. S1C). Consistent with previous studies (Reber et al. 2018), transcription levels of the target gene in the trapped clones were significantly decreased compared with traditional knockout cells (Fig. 1E). These results showed that HIT-trap donors can be effectively inserted into the target loci and disrupt endogenous genes in mESCs.

\section{Systematic assessment of HIT-trapping method in various mammalian cells}

To investigate the general applicability of the HIT-trapping strategy, we developed a defined workflow and applied it to targettrap Hprt, Sall4, and Tet1 in mESCs; ACTB and HPRT1 in human embryonic kidney 293T and melanoma A375 cells; and ROSA26 and THY1 in porcine fetal fibroblasts (PFFs), all of which are expressed in the indicated cell types. Because the successful insertion of the gene-trap cassette results in the expression of GFP driven by the promoter of the trapped gene, the targeted-trapping events could be directly quantified via flow cytometry. Three days after cotransfection with HIT-trap-1C/Cas9/genome-sgRNA plasmid mixtures, the cells were subjected to puromycin selection for $5 \mathrm{~d}$ to enrich targeted-trapping events, followed by expansion for an additional $3 \mathrm{~d}$ for further analysis (Fig. 2A). Flow cytometry results showed that antibiotic selection cultures contained a high percentage of GFPpositive cells in all of the HIT-trapping experiments, ranging from $22.2 \%-68.4 \%$, whereas fewer GFP-positive cells were detected in the control experiments (without genome-sgRNAs) (Fig. 2B; Supplemental Fig. S3). We did not detect any targeted insertion event in the control experiments (Supplemental Fig. S4), suggesting that the puromycin-resistant cells were produced by random integrations via spontaneous NHEJ rather than Cas9-induced NHEJ. The probability of spontaneous integration of the gene-trap vector into a transcribed site across the genome was low, resulting in a lower percentage of GFP-positive cells after selection. Moreover, in the absence of genome-sgRNAs, the number of puromycin-resistant cells was significantly less than that in the HIT-trapping experiments (Supplemental Fig. S5), indicating that the actual knockins occurred at a much higher frequency than nonspecific integrations. As NHEJ-mediated knock-in is nondirectional, the reverse direction knock-in could not generate green fluorescent signals, so the overall knock-in efficiency of HIT-trapping is theoretically much higher than that measured by flow cytometry.

We next sought to determine the fidelity of donor insertion at the target sites and analyzed the junctions generated by HIT-trapping in Hprt of mESCs, ACTB of human A375 cells, and THY1 of PFFs, respectively. Sequence analyses of these genome-donor junctions showed a high level of seamless junction repair (75.4\%-92.4\%) in A375 cells and PFFs, a relatively lower degree of seamless junctions (32.3\%-36.1\%), and an appreciable level of microhomology-mediated end joining (MMEJ) repair-induced deletions (16.4\%-26.2\%) in mESCs (Fig. 2C; Supplemental Fig. S6). 
A
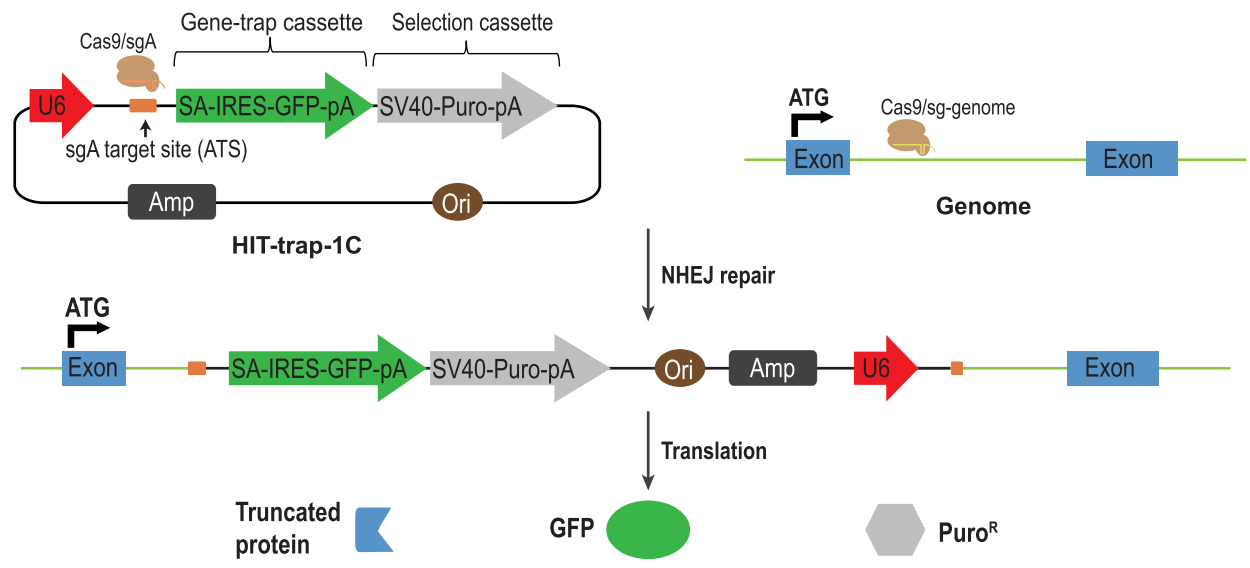

B

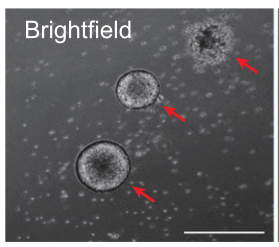

D
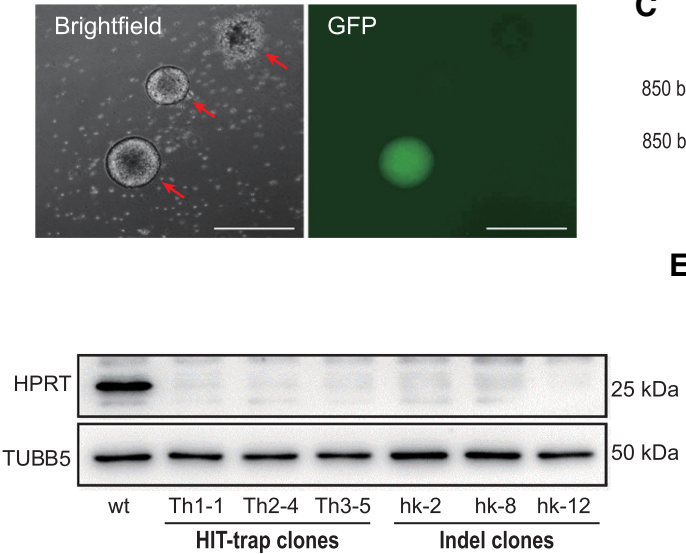

C

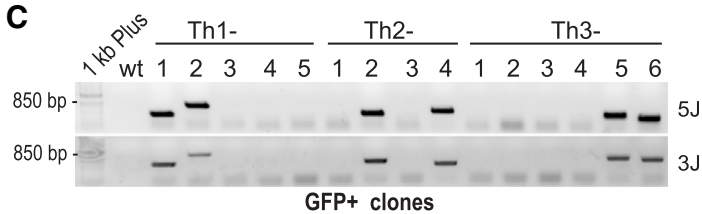

E

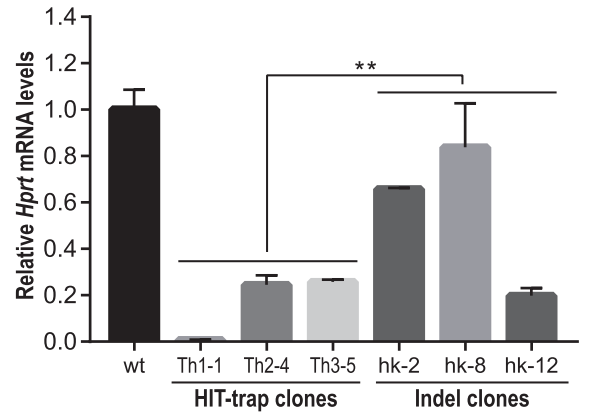

Figure 1. Insertional mutagenesis induced by HIT-trapping in mESCs. (A) Schematic of the HIT-trapping strategy. Concurrent cleavage of the HIT-trap donor and genome by Cas9 RNPs results in targeted trapping. After integration, the gene-trap cassette leads to the expression of a truncated protein and GFP by the promoter of the target gene. The selection cassette expresses a puromycin-resistance gene by a constitutive SV40 promoter. (ATS sequence) GGTATGTCGGGAACCTCTCCAGG; (SA) splice acceptor; (IRES) internal ribosome entry site; (pA) polyadenylation signal. (B) Representative microscopic images of mESC clones after puromycin selection in HIT-trapping. Red arrows indicate apoptotic clone (top), GFP-negative surviving clone (middle), and GFP-positive surviving clone (bottom), respectively. Scale bar, $50 \mu \mathrm{m}$. (C) PCR genotyping of GFP-positive clones confirmed the correct integration of HIT-trap donor at Hprt locus. 5/3], 5'/3' junction. (D,E) Western blot and qPCR analysis of HIT-trap clones targeting the Hprt locus (Th1-1, Th2-4, and Th3-5), with TUBB5 as the loading control. Error bars, SD from three technical replicates. Significance was calculated using the Student's unpaired $t$-test: $(* *) P<0.01$

In considering the possibility that large deletions might disrupt the function of the gene-trap cassette, we summarized our sequencing data and found that most $(91.0 \%)$ of the deletions across the junctions were $<50 \mathrm{bp}$ (Fig. 2D). Because these small deletions did not impair the function of our gene-trap cassette, the vast majority of target-trapped cells produced via HIT-trapping were suitable for downstream applications.

To assess the off-target insertions caused by Cas9, we examined the top three off-target sites predicted by CCTop (Stemmer et al. 2015) for the universal sgA and for each of the genomesgRNAs (Supplemental Table S2). For each off-target site, we performed PCR analyses for both forward and reverse donor integration and observed marginal donor integrations within offtarget loci compared with those within target loci (Supplemental Fig. S7), indicating that the unwanted insertions in puromycinenriched populations were mainly caused by random donor integrations rather than by specific insertions at off-target sites.
Collectively, these results indicated that CRISPR-Cas9-assisted HIT-trapping system is an efficient and precise method to generate gene-trap alleles in mammalian cells.

\section{Various knock-in patterns produced by a two-cut HIT-trap donor}

In our initial experiments, the bacterial backbone and an active RNU6-1 promoter were inserted into the target locus along with the gene-trap, potentially causing unwanted epigenetic modification at the target locus. After genome integration, the sgRNA targeting the vector itself might still be produced. Moreover, potential cryptic splice sites within the vector backbone, leading to aberrant splicing events, would confound functional studies of trapped genes. To avoid these limitations, we constructed two additional two-cut HIT-trap donors (HIT-trap-2C and HIT-trappA2C) that carry identical sgA-targeting sites flanking the insertion part (Supplemental Fig. S8A). Unlike HIT-trap-2C, HIT-trap-

\section{Genome Research}

www.genome.org 
A

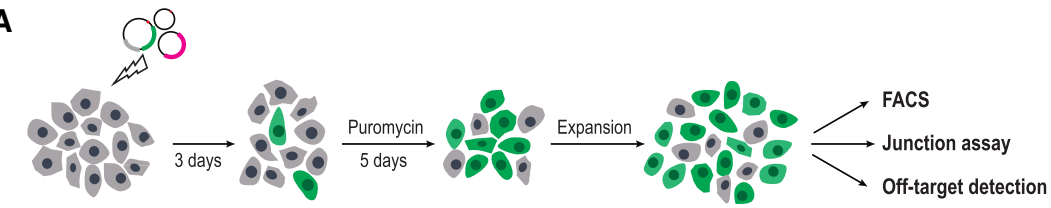

B

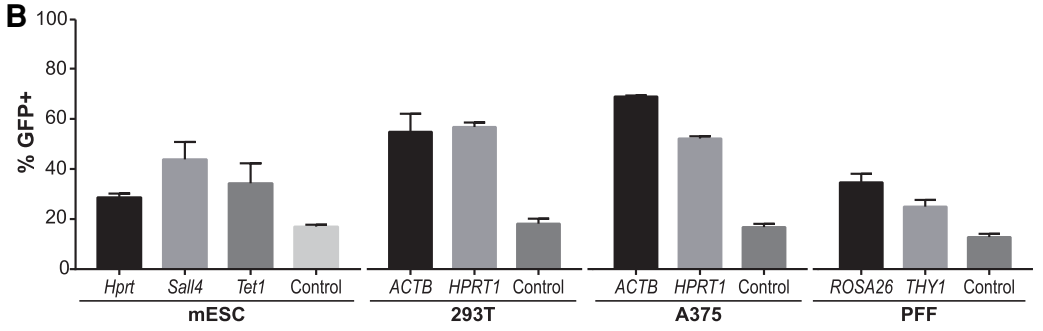

C

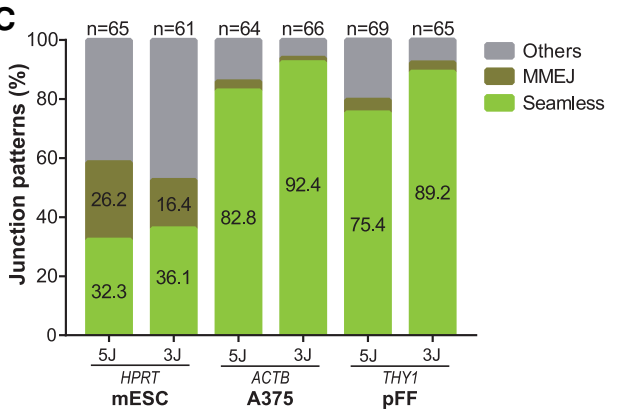

Figure 2. Systematic assessment of the HIT-trapping method in mammalian cells. ( $A$ ) Flowchart of the experimental design. (B) HIT-trapping efficiencies were measured by the percentage of GFP-positive cells among puromycin-resistant cells. As controls, plasmid mixtures without gene-specific sgRNA expressing vectors were transfected into the cells. These results were derived from at least two independent experiments, shown as mean \pm SD. (C) Frequencies and ratios of seamless, microhomology-mediated end joining (MMEJ), and other integration junctions at the target loci. (n) Number of the amplicons analyzed; $(5 / 3]) 5^{\prime} / 3^{\prime}$ junction. (D) Summary statistics of the deletion sizes from junction sequencing. Sequencing data are presented in Supplemental Figure $\mathbf{S 6 .}$

pA2C contains a poly(A)-trap cassette in which a constitutive promoter drives the expression of a puromycin-resistance gene lacking a poly(A) signal (Niwa et al. 1993; Shigeoka et al. 2005). These two donors yielded a comparable frequency of GFP-positive cells to that achieved by the single-cut donor (HIT-trap-1C) in mESCs (Supplemental Fig. S8B,C).

To comprehensively examine the effect of the two-cut donors, it was necessary to check the genome-donor junctions in clonal cell lines. Results showed a high frequency $(41.7 \%, 5 / 12)$ of desired integrations of donor HIT-trap-2C within Hprt of GFPpositive and 6-TG-resistant mESC clones (Supplemental Fig. S9). However, this high frequency seemed largely attributable to preselection by known phenotypes (puromycin/6-TG resistance and GFP positive) of trapping Hprt. Next, we aimed to examine whether transcriptionally silent genes, Nes and Olig2, can be efficiently trapped with HIT-trap-pA2C. Poly(A) trapping captures an endogenous poly(A) signal with which to stabilize the mRNA of the selectable marker gene via a splice donor signal (Niwa et al. 1993; Ishida and Leder 1999). Antibiotic selection should occur only when the poly(A) trap integrates in the forward orientation. Using HIT-trap-pA2C as our knock-in donor, unbiased screening of the puromycin-resistant clones showed high-frequency donor integration at the target loci (15/24 in Nes and 6/24 in Olig2). However, only a few clones showed correct junctions on both sides (two in Nes and one in Olig2) (Fig. 3A). We hypothesized that these undesired outcomes may be because of incomplete cleavage of the donor plasmid. Theoretically, when the donor is linearized at either sgA target site in vivo, it might be immediately captured by the Cas9-induced genomic DSB. Therefore the two-cut donor may yield three knock-in patterns at the target site (Fig. 3B). To test our hypothesis, PCR amplification of genome-donor-specific junctions was performed on these clones. As expected, our results confirmed the existence of all of the three knock-in patterns (Fig. 3C,D). Because of the nature of NHEJ-mediated insertion, the various knock-in patterns should occur at a comparable frequency. Moreover, for the nondirectional promoter HIT-trap donor such as HIT-trap2C, six different knock-in patterns could be generated for one allele. Therefore, the various integration patterns of a two-cut HIT-trap donor would compromise the efficiency of desired insertions and complicate the characterization of target loci.

\section{Optimization of the HIT-trapping method}

To efficiently achieve desired knock-in patterns, we designed several strategies to optimize the donor for HIT-trapping. As shown in Figure 4A, Donor-P was generated by PCR using $5^{\prime}$ phosphorylated primers and only contained the integration components. Donor-X1 was linearized by a restriction enzyme, cutting the $3^{\prime}$ site of the knock-in cassette in vitro instead of in vivo. Donor-X2 was also a linear vector, but the unwanted part of the plasmid donor was completely removed in vitro by restriction enzymes and gel electrophoresis. Donor-LigX2 was a circular vector generated from the self-ligation of Donor-X2 via T4 ligase. All of these donors contained only one sgA target site and were derived from the same plasmid vector HIT-trap-pA1C. These optimized donors were used to target the Nes gene in mESCs to determine the efficiency of the desired knock-in. After electroporation and puromycin selection, survival clones were randomly isolated and genotyped. PCR results showed that desired knockin events were achieved by every optimized donor except Donor-X1, with Donor-LigX2 having the highest frequency (Fig. 4B; Supplemental Fig. S10). This result may be attributed to higher electroporation efficiency and fewer random integrations of the circular donor compared with the linear ones. Therefore, in vitro optimized donors similar to Donor-LigX2 were used for subsequent HIT-trapping experiments.

\section{Simultaneous generation of reversible and conditional knockouts by HIT-trapping}

The nondirectional nature of NHEJ-mediated knock-in using the HIT-promoter trap donor resulted in comparable yields of genetrap incorporated alleles in both desired and undesired orientations (forward and reverse). Previous studies have proven that 
A

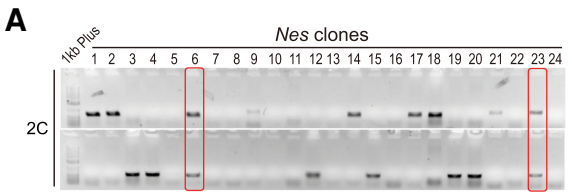

B

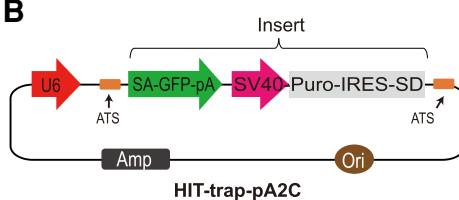

C

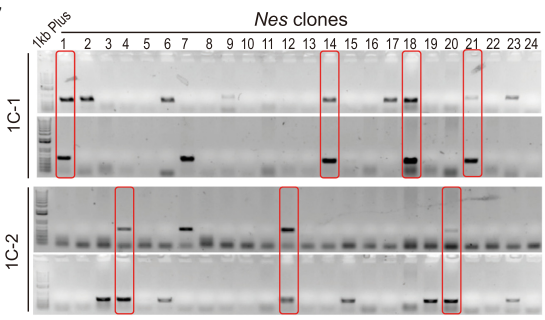

D

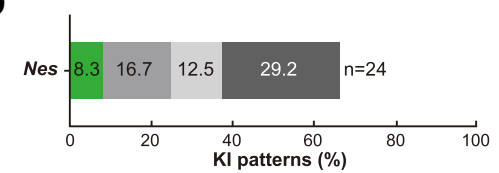

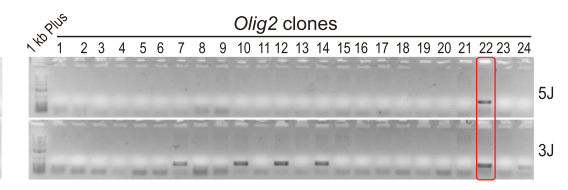

$2 \mathrm{C}$

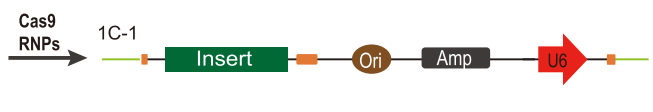

$1 \mathrm{C}-2$
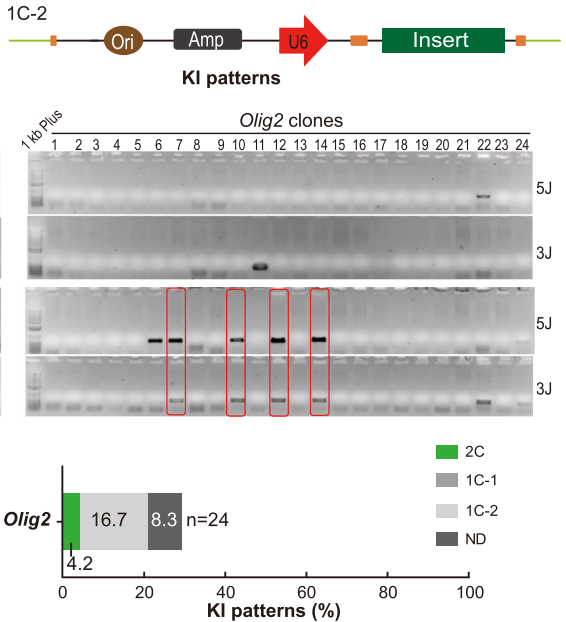

Figure 3. Various knock-in patterns generated by a two-cut HIT-trap donor. (A) PCR genotyping of the puromycin-resistant clones by targeted trapping of Nes and Olig 2 in mESCs. The lanes in red rectangles indicate desired $2 \mathrm{C}$ knock-in patterns. (B) Schematic showing the desired and alternative knock-in patterns by a two-cut donor (HIT-trap-pA2C). SV40-Puro-IRES-SD (poly(A)-trap cassette), SV40 promoter driving expression of a puromycin-resistance gene followed by sequences of an internal ribosome entry site (IRES) and a splice donor (SD). (C) PCR genotyping verified the predicted alternative knock-in patterns (1C-1 and 1C-2) at the Nes and Olig2 loci in mESCs. The lanes in red rectangles indicate the defined alternative knock-in pattern. (5/3]) $5^{\prime} / 3^{\prime}$ junction. (D) Distribution of different knock-in patterns by HITtrap-PA2C. (n) Number of clones checked by PCR; (ND) not determined.

inverted insertion of gene-trap cassette is innocuous and that switching the orientation of the gene-trap cassette via site-specific recombination permits conditional control of the gene function (Schnütgen et al. 2005; Xin 2005; Ni et al. 2012; Economides et al. 2013). Thus, equipping the HIT-trap donor with an invertible mechanism would fully use all of the insertions and generate conditional knockouts. To this end, we constructed a novel HIT-trap vector, HIT-trap-FlEx (Fig. 5A), which is based on the well-characterized strategy termed flip-excision (FIEx) (Schnütgen et al. 2003; Atasoy et al. 2008). Its design is similar to HIT-trap-1C, except that the gene-trap and selection cassette are flanked by heterotypic lox sites (loxP/lox2272). After isolating the knock-in element with PvuI, the purified construct is self-ligated to generate a circular HIT-trap donor LigFV3 for HIT-trapping experiments. Upon electroporation of LigFV3 and Cas9/sgRNAs into cells, multiple cell lines bearing donor integration in either the forward or reverse orientation can be produced simultaneously. After exposure of the gene-trap lines to Cre, step-wise recombination would allow stable inversion of the trap cassette and excision of the selection cassette, thereby switching the mutant alleles to neutral states or the harmless alleles to loss-of-function mutations. Therefore, in combination with nondirectional NHEJ-mediated knock-in, this HITtrapping strategy simultaneously yields both reversible and conditional mutagenesis alleles, making all of the trapped clones usable for further experiments regardless of the starting insertion orientation (Fig. 5B).

Next, we chose to target Nes and Hprt in mESCs and ACTB in A375 cells using donor LigFV3. After puromycin selection, individual colonies were isolated and subjected to PCR genotyping. For actively expressed genes (Hprt and ACTB), the orientation of donor integration could be distinguished by GFP expression directly, allowing forward and reverse insertions to be screened from GFP-positive and -negative clones, respectively. For the silent gene Nes, both forward and reverse insertions needed to be analyzed for each clone. Junction PCR and DNA sequencing revealed that in all of the target loci, cells containing donor insertions in either direction could be obtained by screening a limited number of clones (up to 16) (Fig. 5C; Supplemental Fig. S11). By selecting GFP-positive clones among puromycinresistant ones, it was much easier to obtain forward donor insertions for active genes. These data also show that our optimization of HIT-trapping was simple and effective at avoiding integration of the plasmid backbone.

Western blots showed that the forward LigFV3 integration efficiently abolished the protein expression of Hprt and $A C T B$, whereas the reverse integration did not (Supplemental Fig. S12). Next, to test for recombinase-mediated inversions of the insertions, we selected GFPpositive (clone AG1: trapping $A C T B$ in A375 cells; clone H10: trapping Hprt in mESCs) and GFP-negative clones (clone AN3: targeting ACTB in A375 cells; clone H17: targeting Hprt in mESCs), which represent forward and reverse insertions, respectively. These clones were then transiently transfected with a Cre-expressing plasmid, and as shown in Figure 5D, stable GFP-negative and GFP-positive subclones indicated the unidirectional inversion of the gene-trap cassette. The inversions at the Hprt locus were further confirmed by allele-specific PCR and sequencing (Supplemental Fig. S13) in combination with western blotting (Fig. 5E). As expected, after 6TG and HAT treatment with clones $\mathrm{H} 10$ and H17, their inverted progeny showed corresponding drug resistance associated with the function of $\mathrm{Hprt}$, showing reversible and conditional knockout phenotypes (Fig. 5F).

\section{Highly efficient generation of target-trap alleles in PFFs}

As porcine ESCs remain unavailable to date, SCNT using PFFs is the primary method to generate gene-modified pigs (Tan et al. 2016). Conventional gene targeting approaches are extremely inefficient in PFFs, impeding the production of pig models. To explore whether our HIT-trapping approach could produce stable knock-in PFF clones at high efficiency, we set out to target 21 disease-related genes (Supplemental Table S3). As the gene-trap donor LigFV3 was generic, we only needed to construct appropriate gene-specific

\section{Genome Research}

www.genome.org 
A
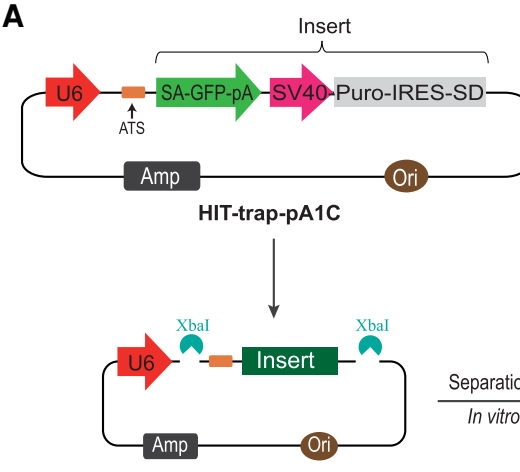

$\frac{\text { Separation }}{\text { In vitro }}$

B

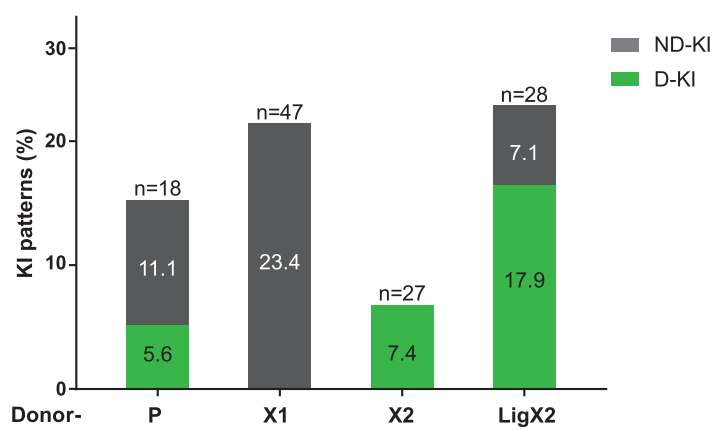

Figure 4. Optimization of the donor for HIT-trapping. (A) Diagrams showing strategies to optimize the HIT-trap donor. All of the optimized donors were derived from the same plasmid donor (HIT-trap-pA1C). Donor-P was PCR generated. Donor-X1 was generated by linearization of the plasmid using Sbfl or EcoRV. Donor-X2 only contains the insertion part of the plasmid, with the backbone removed in vitro using Xbal. Donor-Ligx2 was produced by self-ligation of Donor X2 via T4 ligase. $(B)$ The frequencies of desired/not desired knock-in (D/ND-KI) patterns by the respective optimized donor. ( $n$ ) Number of clones checked by PCR. The genotyping data are presented in Supplemental Figure S10.

pression levels in the heterozygous knockout clones appear to be well below $50 \%$, which could be because of normal variation among different loci or allelespecific expression. These results show that our HIT-trapping system is an effective approach for targeted trapping of genes in PFFs. The preserved modified clones may be ideal resources for cloning pig models for human diseases.

\section{Discussion}

In this study, we devised a HIT-trapping strategy that harnesses the NHEJ repair mechanism for targeted insertion of gene-trap vectors. Compared with conventional gene trapping and targeted trapping, it avoids the laborious site-specific donor construction steps and can be theoretically applied to any cell type. Apart from that, it is also effective regardless of the expressing state of target genes. By using a generic donor coupled with the FlEx switch, we simultaneously obtained reversible and conditional knockouts for user-defined genes, which is unfeasible with existing methods. Possible applications could be microinjection of HITtrap donors and Cas9 RNPs into zygotes.

CRISPR-Cas 9 provides a convenient system for disruption of gene function in mammalian cells. A common gene knockout strategy is to introduce random indels into the exon of the gene of inter-

sgRNA-expressing vectors. PFFs were transfected with the LigFV3/Cas9/sgA/sg-genome mixture and plated at limiting dilutions for clonal isolation. To maintain potential for SCNT, only the well-grown, nonsenescent clones were selected and subjected to genotyping. For each clone, both forward and reverse insertions were detected (a representative example of the PCR screen for PPARG is shown in Fig. 6A). Site-specific junctions were verified by Sanger sequencing (Fig. 6B).

We were able to obtain clones bearing the modified alleles with gene-traps for all of the target sites. The knock-in efficiencies of HIT-trap donors varied from $18.2 \%$ to $82.1 \%$, with an average of $53.2 \%$ (Table 1), regardless of the insertion orientation. A significant portion ( 60 of $189,31.7 \%$ ) of modified clones were produced by biallelic insertions of gene-trap donors in both orientations (Supplemental Fig. S14), suggesting the robustness of HIT-trapping in PFFs. Consistent with junction analysis of enriched cells, junction sequencing also showed a high fraction $(76.1 \%, 118 / 155)$ of the seamless insertion of the gene-trap vector in PFFs. The vast majority $(94.8 \%, 147 / 155)$ of indels around the junctions did not disrupt the lox sites within the donor (Supplemental Fig. S15), reserving the inversion ability of the inserted gene-trap cassettes, so that in most cases, we could achieve both reversible and conditional knockouts by screening only a small number of clones. Moreover, western blots showed decreased protein expression of PPARG, PAX6, LDLR, and PCSK9 in PFF clones heterozygous for the forward HIT-trap integration allele (Supplemental Fig. S16). For PPARG, PAX6, and ACTB (Supplemental Fig. S12), protein ex- est by CRISPR-Cas9-induced NHEJ repair, resulting in coding frameshifts and loss-of-function mutations. However, either the in-frame mutations caused by random indels or incomplete degradation of the corresponding transcript may preserve the function of the target allele. In this context, it can be time-consuming and costly for the identification and isolation of clonal cell lines with desired genetic modifications. In contrast to this random mutation strategy, gene trapping disrupts the transcript of target genes and generates fusion transcripts by the splicing of endogenous exons, leading to predictable loss-of-function alleles. Moreover, HIT-trapping also provides a reporter controlled by the target gene that facilitates analysis of its functions.

To target a specific gene regardless of whether it is expressed, we use a constitutive promoter driving the puromycin-resistance gene within the HIT-trap vector that would also enrich random insertions upon selection. Despite this disadvantage, our technique achieved high rates of targeted trapping at various loci, likely resulting from the robust introduction of DSBs in the genome by Cas9. The efficiency of HIT-trapping depends on the cleavage capacity and accuracy of the sgRNAs against genomic sites. HIT-trapping targets introns within the genome, providing a broader spectrum of available Cas9 target sites and facilitating the choice of sgRNAs with high activity and fidelity.

Previously, single-cut donors were commonly used for NHEJmediated knock-in, which resulted in the incorporation of the entire donor plasmid into the genome (Bachu et al. 2015; Brown et al. 2016; Katoh et al. 2017; Sawatsubashi et al. 2018; Zhang et al. 
A

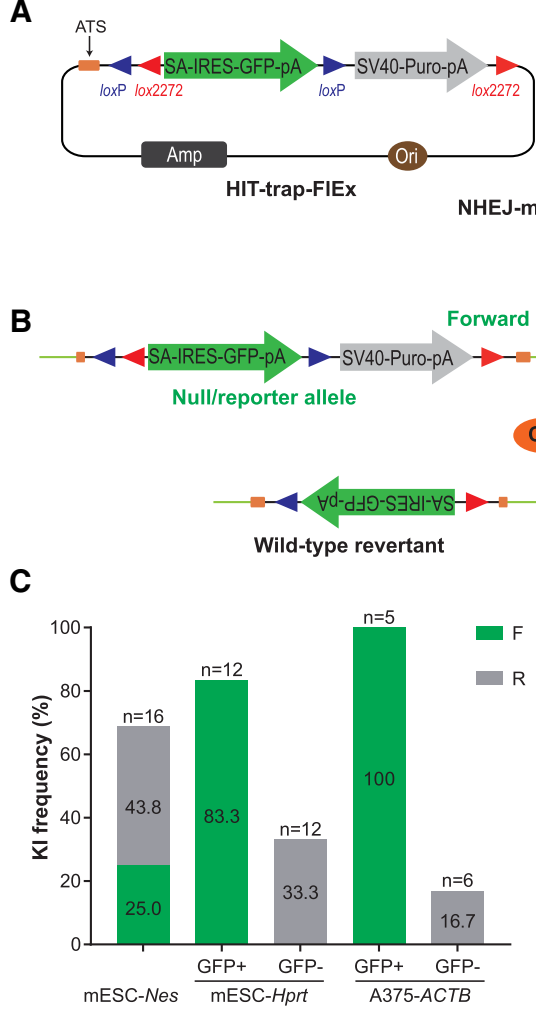

$\mathbf{E}$

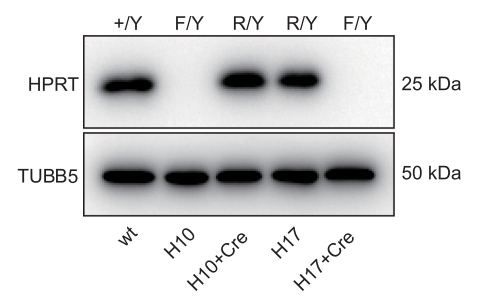

Separation and ligation

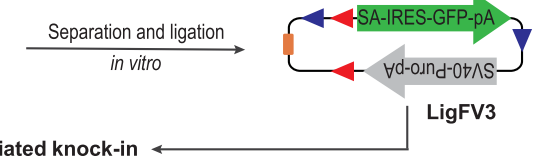

in vivo

$\downarrow$ Reverse

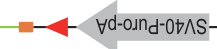

Conditional allele

FIEx switch

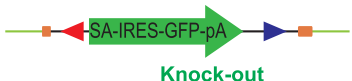

D

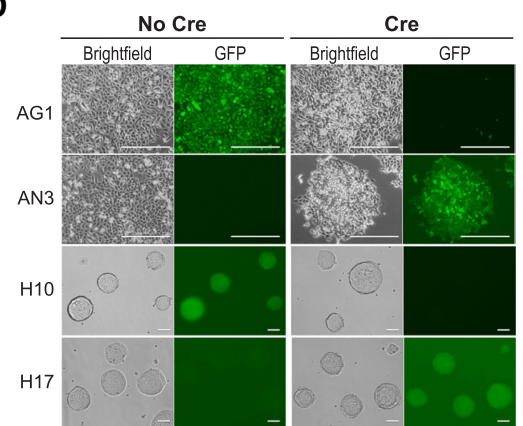

$\mathbf{F}$

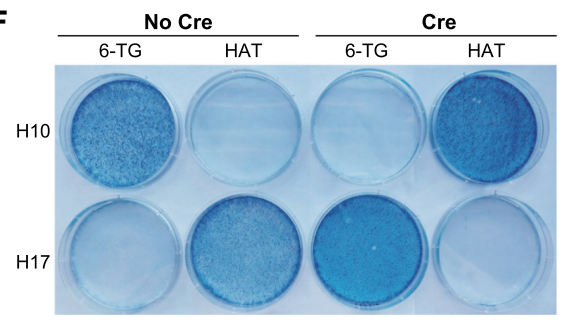

Figure 5. Combining HIT-trapping and FIEx switch enabled the simultaneous generation of reversible and conditional knockouts. $(A, B)$ Schematic showing the workflow and mechanism for simultaneous generation of reversible and conditional knockouts at target sites. Optimized donor LigFV3 was generated through self-ligation of the insertion cassette, excised from the plasmid HIT-trap-FIEx in vitro. Because of the nondirectional feature of NHEJ-mediated knock-in, cells containing either forward or reverse integration can be obtained in a single transfection step. The Cre-mediated FIEx switch enabled the inversion of the gene-trap cassette post insertion, leading to phenotype reverted or conditional knockout. Blue triangles indicate loxP sites; red triangles, lox2272 sites. (C) Knock-in efficiencies of optimized donor LigFV3 at various target sites. (F/R) Forward/reverse integration of the donor; $(n)$ number of screened clones. The genotyping and Sanger sequencing data are presented in Supplemental Figure S11. (D) Images of A375 cells and mESCs bearing FIEx-trap insertions transfected with a Cre expressing plasmid. (AG1/AN3) A375 cell clones with donor insertion at ACTB locus in forward/reverse orientation; $(\mathrm{H} 10 / \mathrm{H} 17)$ mESC clones with donor insertion at Hprt locus in forward/reverse orientation. Scale bar, $50 \mu \mathrm{m}$. (E) Western blot confirmation of the wild-type revertant and conditional knockout phenotypes of Hprt after exposure to Cre in mESCs, with TUBB5 as the loading control. (Y) Y Chromosome (Hprt locus is located on the $X$ Chromosome). ( $F$ ) Methylene blue staining of mESCs mentioned above. mESCs were cultured with 6TG $(2 \mu \mathrm{M})$ or $1 \times$ HAT supplement for $5 \mathrm{~d}$ and were fixed and stained with $0.02 \%$ methylene blue.

2018). Using a two-cut donor flanking the insertion elements with nuclease cleavage sites is a viable option to address this problem for NHEJ-based gene tagging (Lackner et al. 2015; He et al. 2016). Because the target genes for the tagging experiments are highly expressed, it is possible for further enrichment of desired knock-in events through visible fluorescence reporters combined with antibiotic selection. Through unbiased screening and clonal analysis of targeted insertions in silent genes, we show asynchronous cleav- age of two nuclease target sites within the donor plasmid. This event produces three different linearized donors, each competent for NHEJ-mediated end-capture, which results in various insertion patterns that may have been overlooked in previous studies. This phenomenon suggests that in vivo removal of the vector backbone by a two-cut donor is not as straightforward as expected and leads to multiple types of insertions. Without a selectable-marker to pre-eliminate nonspecific insertions (i.e., tagging nonexpressed genes), achieving the desired insertion with two-cut donor-mediated homology-independent knock-in may be challenging. In this study, we developed a simple optimized strategy by removing the plasmid backbone in vitro instead of in vivo. The in vitro modified HIT-trap donors were easy to produce and yielded knock-in efficiency comparable to plasmid donors. This strategy could potentially be adapted to obtain clean insertions of transgenes when using homology-independent knock-in strategies. Alternatively, the HIT-trap donors without the plasmid backbone can be prepared from minicircle DNA vectors.

In agreement with other studies, knock-in of HIT-trap donors was largely mediated by the conventional NHEJ pathway (Bétermier et al. 2014; Geisinger et al. 2016; Schmid-Burgk et al. 2016). We observed high proportions of seamless junctions in A375 cells and PFFs, with a lower frequency of precise repair in mESCs. The degree of precision ligation might be influenced by genetic and cellular context. A major shortcoming of HIT-trapping is that the indels inherent to NHEJ around the junctions may impair the functional elements within the donor. However, in most cases, this risk can be tolerated in intronbased HIT-trapping.

A practical application of HIT-trapping is to introduce mutations in livestock species, and we chose the pig as the focus of this study. Genetically modified pigs are important for agriculture and biomedical studies. Authentic porcine ESCs have still not been isolated, and SCNT using primary fibroblasts remains the most popular method to create genetically modified pigs. However, because of the limited lifespan and low HR activity of primary fibroblasts, genetic modifications are much more difficult to achieve than in immortalized cell types. HIT-trapping, which is independent of ES cells and HDR, provides a solution to circumvent these problems. We report successful implementation of this method for targeting more than 20 loci in PFFs, revealing precise, high-frequency

\section{Genome Research}

www.genome.org 
A
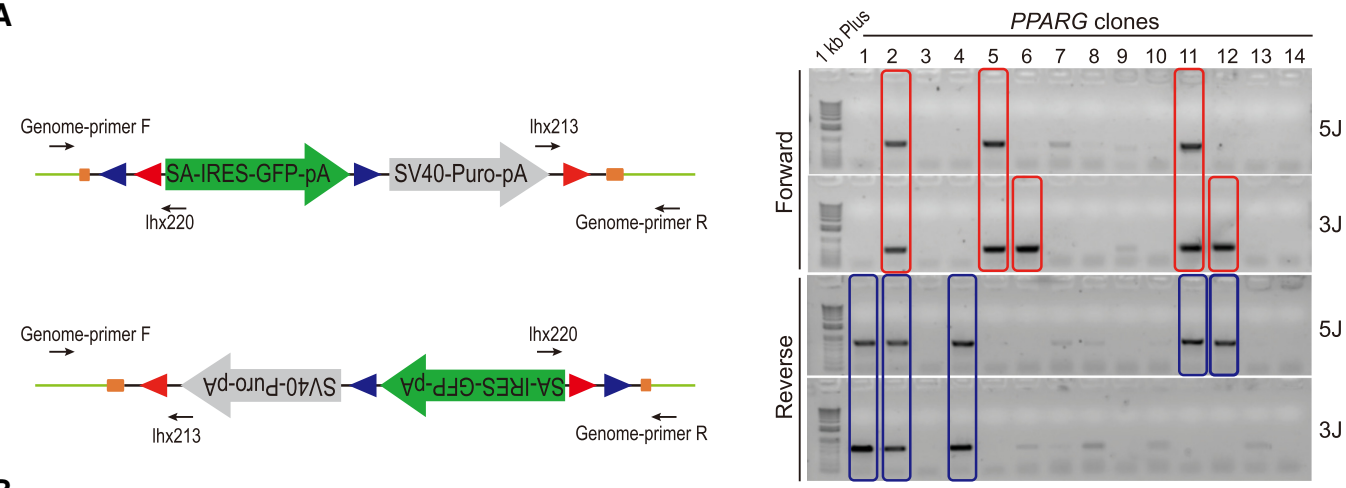

B

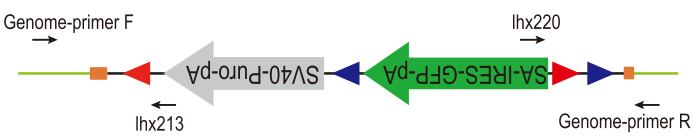

\begin{abstract}
PPARG Forward
Seamless 5 -ggcctcgcggtattaatcac TCCAGGacttcatttctag- $3^{\prime}$

PPARG-2 5'-ggcctcgcggtattaatcacTCCAGGacttcatttctag-3' seamless PPARG-5 5'-ggcctcgcggtattaatcacTCCAGGacttcatttctag-3' seamless PPARG-11 5'-ggcctcgcggtattaatcacTCCAGGacttcatttctag-3' seamless
\end{abstract}

PPARG Reverse

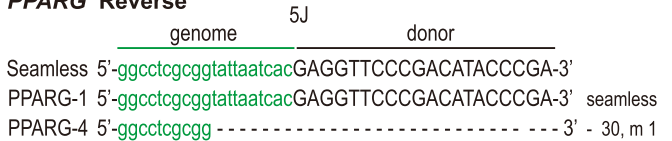

\begin{abstract}
$3 \mathrm{~J}$
Seamless 5'-TCGGGTATGTCGGGACCTCtagtggatggaagacacaca-3

PPARG-2 5'-TCGGGTATGTCGGGAACCTCtagtggatggaagacacaca-3' seamless PPARG-5 5'-TCGGGTATGTCGGGAACCTCtagtggatggaagacacaca-3' seamless PPARG-11 5'-TCGGGTATGTCGGGAACCTCtagtggatggaagacacaca-3' seamless
\end{abstract}

$$
\begin{array}{lll} 
& \frac{\text { donor }}{3} & \multicolumn{1}{c}{\text { genome }} \\
\text { Seamless } & 5^{\prime} \text {-ctagaaaatgaagtCCTGGAtagtggatggaagacacaca-3' } \\
\text { PPARG-1 } & 5^{\prime} \text {-ctagaaaatgaagtCCTGGACtagtggatggaagacacaca-3' + } 1 \\
\text { PPARG-4 } & 5^{\prime} \text {-ctagaaaatgaagtCCTGGAGAnnnnnnnCCCGcaca-3' + 3, m } 16
\end{array}
$$

Figure 6. Generation of PFF clones carrying gene-trap cassettes at the target loci via HIT-trapping. ( $A$ ) Schematic showing PCR genotyping of the screened PFF clones produced via HIT-trapping (left) and representative genotyping results for targeted trapping of the PPARG locus (right). Lhx220/ 213 were donor-specific primers. Lanes in red/blue rectangles indicate positive forward/reverse insertions. (5/3j) $5^{\prime} / 3^{\prime}$ junction. ( $B$ ) Sequence confirmation of gene-trap insertions at the PPARG locus. Genome sequences are shown in green. PAMs are shown in blue. Insertions or mismatches are shown in red. Deletions are shown as dashed lines. (m) Mismatches.

genome editing. Moreover, both reversible and conditional knockout alleles were simultaneously obtained with only limited clone screenings (as few as four clones per gene). These clonal cell lines are ready for future pig SCNT experiments.

In summary, HIT-trapping provides a simple and efficient methodology to introduce reversible and conditional mutations at user-defined loci via CRISPR-Cas9-based NHEJ. The speed and ease of implementing this strategy provides a novel and valuable tool for creating mutant animal models.

\section{Methods}

\section{Plasmid construction}

All gene-specific sgRNAs were chosen through the online tool CHOPCHOP (Montague et al. 2014) and listed in Supplemental Tables S1 and S3. Oligonucleotides for sgRNA templates were synthesized, annealed, and inserted into the BbsI site of pCRISPR-sg6 (Xu et al. 2017). The Cas9-expressing plasmid pM3-Cas9 was constructed by replacing the GFP cassette from pMax-GFP (Lonza) with the Cas9 sequence from pX330 (Addgene 42230).

Various donor plasmids were constructed through restriction enzyme cloning and Gibson assembly (NEB). Briefly, to generate the HIT-trap-1C/2C and HIT-trap-pA1C/2C, the sgA sequence was first inserted into the pX335 (Addgene 42335) via BbsI and then digested with KpnI/EcoRI to remove CMV-Cas9n cassette and inserted various amplicons by Gibson assembly, respectively. We amplified the DNA fragment encoding SA-IRES-GFP/SA-GFP from pZGs (Wu et al. 2007), SV40-puro-pA from pEF1a-GFPPuro, and SD sequence from pFind2. The sgA target site was simultaneously added by primer design or directly synthesized for Gibson assembly. HIT-trap-FlEx was generated by Gibson assem- bly of the SA-IRES-GFP-pA and SV40-puro-pA amplicons on a synthesized backbone containing a sgA target site and loxP/lox2272 sites for FlEx switch. Primers used for plasmid construction are listed in Supplemental Table S4. Annotated vector sequences are provided as Supplemental Files.

\section{Cell culture and transfection}

Mouse ESCs were maintained in 2i/LIF (Silva et al. 2008) on gelatin-coated plates or irradiated feeders. HEK 293T cells, A375 cells, and PFFs were cultured in Dulbecco's Modified Eagle's Medium (DMEM) supplemented with 10\% fetal bovine serum (FBS). For each transfection experiment, about $10^{6}$ cells were mixed with $6 \mu \mathrm{g}$ DNA $(2 \mu \mathrm{g}$ HIT-trap donor, $1 \mu \mathrm{g}$ sgRNA-expressing plasmids, and $3 \mu \mathrm{g}$ Cas9-expressing plasmid) and resuspended in the prewarmed Nucleofector solution. The electroporation was performed with the 4D-Nucleofector system (Lonza), per the manufacturer's protocols. Transfected cells were treated with $1 \mu \mathrm{g} / \mathrm{mL}$ puromycin (Invivogen) for $5 \mathrm{~d}$ to select the knock-in events, and the percentages of GFP-positive cells were measured by a FACS Calibur machine (BD Biosciences).

\section{Genomic DNA isolation and junction analyses}

The genomic DNA was extracted as previously described (Wu et al. 2008). The genome-donor junctions were detected for locus-specific donor integration by PCR (GoTaq Polymerase [Promega] according to manufacturer's instructions). Primers used for amplification of junctions are shown in Supplemental Tables S5 and S6. PCR products of pooled cells were ligated into the pMD19-T vector (TakaRa) via TA cloning and sequenced. PCR products of clonal cell lines were directly purified and sequenced. 
Table 1. Summary of genotyping results of PFF clones screened by HIT-trapping

\begin{tabular}{|c|c|c|c|c|c|c|c|}
\hline \multirow[b]{2}{*}{ Target gene } & \multicolumn{3}{|c|}{ Forward } & \multicolumn{3}{|c|}{ Reverse } & \multirow[b]{2}{*}{$\mathrm{KI}$ frequency $(\%)^{\mathrm{a}}$} \\
\hline & $5^{\prime}$ junction & $3^{\prime}$ junction & $5^{\prime}+3^{\prime}$ junction & $5^{\prime}$ junction & $3^{\prime}$ junction & $5^{\prime}+3^{\prime}$ junction & \\
\hline THY1 & 0 & 2 & 3 & 0 & 0 & 1 & $6 / 14(42.9)$ \\
\hline$L D L R$ & 1 & 1 & 1 & 0 & 0 & 3 & $3 / 6(50.0)$ \\
\hline PCSK9 & 1 & 0 & 2 & 0 & 1 & 0 & $4 / 20(20.0)$ \\
\hline UCP3 & 2 & 4 & 1 & 2 & 2 & 4 & $11 / 24(45.8)$ \\
\hline APOA1 & 0 & 1 & 8 & 1 & 1 & 10 & $13 / 16(81.3)$ \\
\hline APOC 3 & 0 & 0 & 1 & 0 & 0 & 6 & 6/11 (54.6) \\
\hline GLP1R & 2 & 2 & 1 & 0 & 1 & 1 & $6 / 8(75.0)$ \\
\hline PPARG & 0 & 2 & 3 & 2 & 0 & 3 & 7/14 (50.0) \\
\hline HNF1A & 0 & 1 & 4 & 2 & 1 & 3 & $8 / 15(53.3)$ \\
\hline INS & 0 & 0 & 1 & 0 & 0 & 1 & $2 / 4(50.0)$ \\
\hline TP53 & 1 & 11 & 1 & 0 & 0 & 13 & $20 / 26(76.9)$ \\
\hline HNF1B & 1 & 4 & 1 & 0 & 0 & 8 & $10 / 18(55.6)$ \\
\hline PAX6 & 0 & 1 & 6 & 4 & 0 & 1 & $11 / 19(57.9)$ \\
\hline SMN1 & 0 & 3 & 5 & 1 & 1 & 5 & $10 / 24(41.7)$ \\
\hline SMNDC1 & 1 & 6 & 2 & 3 & 1 & 13 & $21 / 31(67.7)$ \\
\hline PKD1 & 2 & 1 & 3 & 1 & 3 & 3 & $11 / 18(61.1)$ \\
\hline RAG2 & 0 & 0 & 1 & 0 & 0 & 1 & $2 / 11(18.2)$ \\
\hline$A R$ & 0 & 6 & 1 & 5 & 0 & 4 & $11 / 16(68.8)$ \\
\hline IL6ST & 1 & 0 & 1 & 0 & 0 & 0 & $2 / 5(40.0)$ \\
\hline$\angle A M B 3$ & 1 & 0 & 1 & 0 & 0 & 0 & $2 / 8(25.0)$ \\
\hline COL17A1 & 0 & 2 & 9 & 1 & 2 & 17 & $23 / 28(82.1)$ \\
\hline
\end{tabular}

${ }^{\mathrm{a} E a c h}$ of the screened clones was genotyped for both forward and reveres insertions. As the diploid feature of PFFs, some clones contained biallelic insertions of gene-trap donors in both orientations. Knock-in (KI) frequency was calculated as (clones with any donor insertion at the target site / clones genotyped). The genotyping and Sanger sequencing data are presented in Figure 6 and Supplemental Figures S14 and S15.

\section{RT-PCR and western blot analyses}

Total RNA was purified using the RNeasy mini kit (Qiagen) and was reverse-transcripted into cDNA using TransScript one-step gDNA removal and cDNA synthesis supermix (TransGen Biotech). RTPCR was performed using green qPCR supermix (TransGen Biotech) and performed on an ABI7500 real-time PCR system. Primers used for RT-PCR are listed in Supplemental Table S7. Cultured cells were harvested and resuspended in RIPA lysis buffer (Beyotime Biotechnology). Western blotting followed using standard protocols. Antibodies are listed in Supplemental Table S8.

\section{Generation of an in vitro modified donor: LigFV3}

Donor LigFV3 was derived from HIT-trap-FIEx as follows: The plasmid DNA was digested overnight with PvuI (NEB) and subjected to gel electrophoresis, excised, and purified to remove the backbone. The purified linear DNA was self-ligated by T4 DNA ligase (NEB) followed by treatment for $10 \mathrm{~min}$ at $65^{\circ} \mathrm{C}$ to inactivate the ligase.

\section{Screening for target-trapped PFF clones}

For each electroporation, $\sim 5 \times 10^{5}$ PFFs were electroporated with corresponding DNA mixture and seeded onto three $100-\mathrm{mm}$ plates. Three days after transfection, the cells were cultured with the medium containing puromycin $(1.0 \mu \mathrm{g} / \mathrm{mL})$ for $5 \mathrm{~d}$; emerging clones were trypsinized, expanded, and screened by PCR and Sanger sequencing.

\section{Competing interest statement}

The authors declare no competing interests.

\section{Acknowledgments}

We thank Dr. Lara Carroll (University of Utah) for the careful reading of the manuscript. This work was supported by the Transgenic Research Grants 2016ZX08009003, the National Natural Science Foundation of China (grant no. 32002180), and the 2020 Research Program of Sanya Yazhou Bay Science and Technology City (grant no. 202002011).

Author contributions: H.L. and S.W. conceived of and designed the experiments. H.L. and J.L. performed most of the experiments with help from Z.G., Y.Y., F.G., and G.C. H.L., T.F., J.L., X.D., and S.W. analyzed the data and wrote the manuscript. H.L and J.L. contributed equally to this work.

\section{References}

Atasoy D, Aponte Y, Su HH, Sternson SM. 2008. A FLEX switch targets channelrhodopsin-2 to multiple cell types for imaging and long-range circuit mapping. J Neurosci 28: 7025-7030. doi:10.1523/JNEUROSCI.1954-08 .2008

Bachu R, Bergareche I, Chasin LA. 2015. CRISPR-Cas targeted plasmid integration into mammalian cells via non-homologous end joining. Biotechnol Bioeng 112: 2154-2162. doi:10.1002/bit.25629

Bétermier M, Bertrand P, Lopez BS. 2014. Is non-homologous end-joining really an inherently error-prone process? PLoS Genet 10: e1004086. doi:10.1371/journal.pgen.1004086

Branda CS, Dymecki SM. 2004. Talking about a revolution: the impact of site-specific recombinases on genetic analyses in mice. Dev Cell 6: 728. doi:10.1016/S15345807(03)00399-X

Brown A, Woods WS, Perez-Pinera P. 2016. Multiplexed targeted genome engineering using a universal nuclease-assisted vector integration system. ACS Synth Biol 5: 582-588. doi:10.1021/acssynbio.6b00056

Chen F, Pruett-Miller SM, Huang Y, Gjoka M, Duda K, Taunton J, Collingwood TN, Frodin M, Davis GD. 2011. High-frequency genome editing using ssDNA oligonucleotides with zinc-finger nucleases. Nat Methods 8: 753-755. doi:10.1038/nmeth.1653

Cristea S, Freyvert Y, Santiago Y, Holmes MC, Urnov FD, Gregory PD, Cost GJ. 2013. In vivo cleavage of transgene donors promotes nuclease-mediated targeted integration. Biotechnol Bioeng 110: 871-880. doi:10.1002/ bit. 24733

\section{Genome Research}

www.genome.org 
Doetschman T, Gregg RG, Maeda N, Hooper ML, Melton DW, Thompson S, Smithies O. 1987. Targetted correction of a mutant HPRT gene in mouse embryonic stem cells. Nature 330: 576-578. doi:10.1038/330576a0

Doudna JA, Charpentier E. 2014. The new frontier of genome engineering with CRISPR-Cas9. Science 346: 1258096. doi:10.1126/science.1258096

Economides AN, Frendewey D, Yang P, Dominguez MG, Dore AT, Lobov IB, Persaud T, Rojas J, McClain J, Lengyel P, et al. 2013. Conditionals by inversion provide a universal method for the generation of conditional alleles. Proc Natl Acad Sci 110: E3179-E3188. doi:10.1073/pnas .1217812110

Friedel RH, Plump A, Lu X, Spilker K, Jolicoeur C, Wong K, Venkatesh TR, Yaron A, Hynes M, Chen B, et al. 2005. From the cover: Gene targeting using a promoterless gene trap vector ("targeted trapping") is an efficient method to mutate a large fraction of genes. Proc Natl Acad Sci 102: $13188-13193$. doi: $10.1073 /$ pnas. 0505474102

Geisinger JM, Turan S, Hernandez S, Spector LP, Calos MP. 2016. In vivo blunt-end cloning through CRISPR/Cas9-facilitated non-homologous end-joining. Nucleic Acids Res 44: e76. doi:10.1093/nar/gkv1542

Gossler A, Joyner A, Rossant J, Skarnes W. 1989. Mouse embryonic stem cells and reporter constructs to detect developmentally regulated genes. Science 244: 463-465. doi:10.1126/science.2497519

He X, Tan C, Wang F, Wang Y, Zhou R, Cui D, You W, Zhao H, Ren J, Feng B. 2016. Knock-in of large reporter genes in human cells via CRISPR/Cas9induced homology-dependent and independent DNA repair. Nucleic Acids Res 44: e85. doi:10.1093/nar/gkw064

Hsu PD, Lander ES, Zhang F. 2014. Development and applications of CRISPR-Cas9 for genome engineering. Cell 157: 1262-1278. doi:10 $.1016 /$ j.cell.2014.05.010

Ishida Y, Leder P. 1999. RET: a poly A-trap retrovirus vector for reversible disruption and expression monitoring of genes in living cells. Nucleic Acids Res 27: e35. doi:10.1093/nar/27.24.e35

Katoh Y, Michisaka S, Nozaki S, Funabashi T, Hirano T, Takei R, Nakayama K. 2017. Practical method for targeted disruption of cilia-related genes by using CRISPR/Cas9-mediated, homology-independent knock-in system. Mol Biol Cell 28: 898-906. doi:10.1091/mbc.E17-01-0051

Lackner DH, Carré A, Guzzardo PM, Banning C, Mangena R, Henley T, Oberndorfer S, Gapp BV, Nijman SMB, Brummelkamp TR, et al. 2015. A generic strategy for CRISPR-Cas9-mediated gene tagging. Nat Commun 6: 10237. doi:10.1038/ncomms10237

Maresca M, Lin VG, Guo N, Yang Y. 2013. Obligate ligation-gated recombination (ObLiGaRe): custom-designed nuclease-mediated targeted integration through nonhomologous end joining. Genome Res 23: 539546. doi:10.1101/gr.145441.112

Mehravar M, Shirazi A, Nazari M, Banan M. 2019. Mosaicism in CRISPR/ Cas9-mediated genome editing. Dev Biol 445: 156-162. doi:10.1016/j .ydbio.2018.10.008

Montague TG, Cruz JM, Gagnon JA, Church GM, Valen E. 2014. CHOPCHOP: a CRISPR/Cas9 and TALEN web tool for genome editing. Nucleic Acids Res 42: W401-W407. doi:10.1093/nar/gku410

Ni TT, Lu J, Zhu M, Maddison LA, Boyd KL, Huskey L, Ju B, Hesselson D, Zhong TP, Page-McCaw PS, et al. 2012. Conditional control of gene function by an invertible gene trap in zebrafish. Proc Natl Acad Sci 109: 15389-15394. doi:10.1073/pnas.1206131109

Niwa H, Araki K, Kimura S, Taniguchi S, Wakasugi S, Yamamura K. 1993. An efficient gene-trap method using poly A trap vectors and characterization of gene-trap Events1. J Biochem 113: 343-349. doi:10.1093/oxford journals.jbchem.a124049

Orlando SJ, Santiago Y, DeKelver RC, Freyvert Y, Boydston EA, Moehle EA, Choi VM, Gopalan SM, Lou JF, Li J, et al. 2010. Zinc-finger nucleasedriven targeted integration into mammalian genomes using donors with limited chromosomal homology. Nucleic Acids Res 38: e152. doi:10.1093/nar/gkq512

Porteus MH, Carroll D. 2005. Gene targeting using zinc finger nucleases. Nat Biotechnol 23: 967-973. doi:10.1038/nbt1125

Quadros RM, Miura H, Harms DW, Akatsuka H, Sato T, Aida T, Redder R, Richardson GP, Inagaki Y, Sakai D, et al. 2017. Easi-CRISPR: a robust method for one-step generation of mice carrying conditional and insertion alleles using long ssDNA donors and CRISPR ribonucleoproteins. Genome Biol 18: 92. doi:10.1186/s13059-017-1220-4
Ran FA, Hsu PD, Wright J, Agarwala V, Scott DA, Zhang F. 2013. Genome engineering using the CRISPR-Cas9 system. Nat Protoc 8: 2281-2308. doi:10.1038/nprot.2013.143

Reber S, Mechtersheimer J, Nasif S, Benitez JA, Colombo M, Domanski M, Jutzi D, Hedlund E, Ruepp M-D. 2018. CRISPR-Trap: a clean approach for the generation of gene knockouts and gene replacements in human cells. Mol Biol Cell 29: 75-83. doi:10.1091/mbc.E17-05-0288

Reddy P, Vilella F, Izpisua Belmonte JC, Simón C. 2020. Use of customizable nucleases for gene editing and other novel applications. Genes (Basel) 11: 976 . doi: $10.3390 /$ genes 11090976

Sawatsubashi S, Joko Y, Fukumoto S, Matsumoto T, Sugano SS. 2018 Development of versatile non-homologous end joining-based knockin module for genome editing. Sci Rep 8: 593. doi:10.1038/s41598017-18911-9

Schmid-Burgk JL, Höning K, Ebert TS, Hornung V. 2016. CRISPaint allows modular base-specific gene tagging using a ligase-4-dependent mechanism. Nat Commun 7: 12338. doi:10.1038/ncomms12338

Schnütgen F, Doerflinger N, Calléja C, Wendling O, Chambon P, Ghyselinck NB. 2003. A directional strategy for monitoring Cre-mediated recombination at the cellular level in the mouse. Nat Biotechnol 21: 562-565. doi:10.1038/nbt811

Schnütgen F, De-Zolt S, Van Sloun P, Hollatz M, Floss T, Hansen J, Altschmied J, Seisenberger C, Ghyselinck NB, Ruiz P, et al. 2005. Genomewide production of multipurpose alleles for the functional analysis of the mouse genome. Proc Natl Acad Sci 102: 7221-7226. doi:10.1073/pnas.0502273102

Shigeoka T, Kawaichi M, Ishida Y. 2005. Suppression of nonsense-mediated mRNA decay permits unbiased gene trapping in mouse embryonic stem cells. Nucleic Acids Res 33: e20. doi:10.1093/nar/gni022

Silva J, Barrandon O, Nichols J, Kawaguchi J, Theunissen TW, Smith A. 2008. Promotion of reprogramming to ground state pluripotency by signal inhibition. PLoS Biol 6: e253. doi:10.1371/journal.pbio.0060253

Skarnes W, von Melchner H, Wurst W, Hicks G, Nord A, Cox T, Young S, Ruiz P, Soriano P, Tessier-Lavigne M, et al. 2004. A public gene trap resource for mouse functional genomics. Nat Genet 36: 543-544. doi:10 .1038/ng0604-543

Stanford WL, Cohn JB, Cordes SP. 2001. Gene-trap mutagenesis: past, present and beyond. Nat Rev Genet 2: 756-768. doi:10.1038/35093548

Stemmer M, Thumberger T, del Sol Keyer M, Wittbrodt J, Mateo JL. 2015. CCTop: an intuitive, flexible and reliable CRISPR/Cas9 target prediction tool. PLoS One 10: e0124633. doi:10.1371/journal.pone.0124633

Suzuki K, Tsunekawa Y, Hernandez-Benitez R, Wu J, Zhu J, Kim EJ, Hatanaka F, Yamamoto M, Araoka T, Li Z, et al. 2016. In vivo genome editing via CRISPR/Cas9 mediated homology-independent targeted integration. Nature 540: 144-149. doi:10.1038/nature20565

Tan W, Proudfoot C, Lillico SG, Whitelaw CBA. 2016. Gene targeting, genome editing: from dolly to editors. Transgenic Res 25: 273-287. doi:10.1007/s11248-016-9932-x

Wu S, Ying G, Wu Q, Capecchi MR. 2007. Toward simpler and faster genome-wide mutagenesis in mice. Nat Genet 39: 922-930. doi:10.1038/ ng2060

Wu S, Ying G, Wu Q, Capecchi MR. 2008. A protocol for constructing gene targeting vectors: generating knockout mice for the cadherin family and beyond. Nat Protoc 3: 1056-1076. doi:10.1038/nprot.2008.70

Xin H-B. 2005. Gene trap and gene inversion methods for conditional gene inactivation in the mouse. Nucleic Acids Res 33: e14. doi:10.1093/nar/ gni016

Xu C, Qi X, Du X, Zou H, Gao F, Feng T, Lu H, Li S, An X, Zhang L, et al. 2017. piggyBac mediates efficient in vivo CRISPR library screening for tumorigenesis in mice. Proc Natl Acad Sci 114: 722-727. doi:10.1073/pnas .1615735114

Zhang C, He X, Kwok YK, Wang F, Xue J, Zhao H, Suen KW, Wang CC, Ren J, Chen GG, et al. 2018. Homology-independent multiallelic disruption via CRISPR/Cas9-based knock-in yields distinct functional outcomes in human cells. BMC Biol 16: 151. doi:10.1186/s12915-018-0616-2

Received September 3, 2020; accepted in revised form March 30, 2021. 


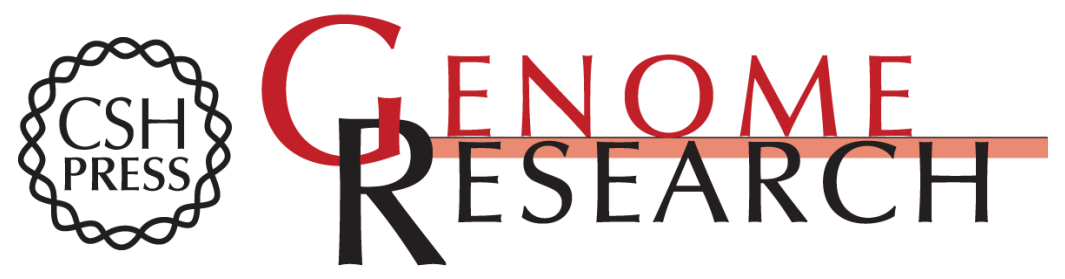

\section{A HIT-trapping strategy for rapid generation of reversible and conditional alleles using a universal donor}

Hengxing Lu, Jun Liu, Tao Feng, et al.

Genome Res. 2021 31: 900-909 originally published online April 1, 2021

Access the most recent version at doi:10.1101/gr.271312.120

Supplemental Material

References

Creative

Commons

License

Email Alerting

Service
http://genome.cshlp.org/content/suppl/2021/04/20/gr.271312.120.DC1

This article cites 45 articles, 11 of which can be accessed free at: http://genome.cshlp.org/content/31/5/900.full.html\#ref-list-1

This article is distributed exclusively by Cold Spring Harbor Laboratory Press for the first six months after the full-issue publication date (see

https://genome.cshlp.org/site/misc/terms.xhtml). After six months, it is available under a Creative Commons License (Attribution-NonCommercial 4.0 International), as described at http://creativecommons.org/licenses/by-nc/4.0/.

Receive free email alerts when new articles cite this article - sign up in the box at the top right corner of the article or click here.

\section{Affordable, Accurate Sequencing.}

To subscribe to Genome Research go to:

https://genome.cshlp.org/subscriptions 